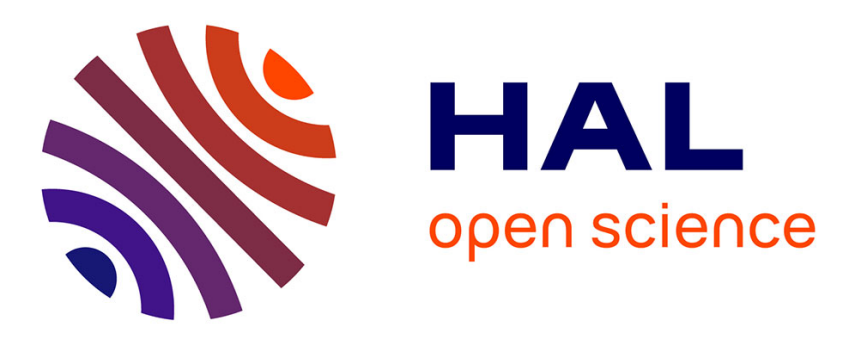

\title{
Exploring the spatial precision of focal infrared neural stimulation in the cortex of GCaMP6f mice
}

Attila Kaszas, Ivo Vanzetta, Gergely Szalay, Alexandra Bojdan, Balazs Rozsa, Rodney O'Connor, David Moreau

\section{- To cite this version:}

Attila Kaszas, Ivo Vanzetta, Gergely Szalay, Alexandra Bojdan, Balazs Rozsa, et al.. Exploring the spatial precision of focal infrared neural stimulation in the cortex of GCaMP6f mice. Optogenetics and Optical Manipulation 2020, Feb 2020, San Francisco, United States. pp.29, 10.1117/12.2551479 . hal-03065188

\section{HAL Id: hal-03065188 \\ https://hal.science/hal-03065188}

Submitted on 15 Dec 2020

HAL is a multi-disciplinary open access archive for the deposit and dissemination of scientific research documents, whether they are published or not. The documents may come from teaching and research institutions in France or abroad, or from public or private research centers.
L'archive ouverte pluridisciplinaire HAL, est destinée au dépôt et à la diffusion de documents scientifiques de niveau recherche, publiés ou non, émanant des établissements d'enseignement et de recherche français ou étrangers, des laboratoires publics ou privés. 


\title{
Exploring the spatial precision of focal infrared neural stimulation in the cortex of GCaMP6f mice
}

\author{
Attila Kaszas ${ }^{\mathrm{a}}$, Ivo Vanzetta ${ }^{\mathrm{a}}$, Gergely Szalay ${ }^{\mathrm{b}}$, Alexandra Bojdán ${ }^{\mathrm{b}}$, Balázs Rózsa ${ }^{\mathrm{b}, \mathrm{c}}$, Rodney \\ O'Connor $^{\text {a,d }}$, David Moreau*d \\ ${ }^{a}$ Institut de Neurosciences de la Timone, CNRS UMR 7289 \& Aix- Marseille Université 13005 \\ Marseille, France; ${ }^{b}$ Laboratory of 3D Functional Network and Dendritic Imaging, Institute of \\ Experimental Medicine, Budapest 1083, Hungary; ${ }^{\mathrm{c} T w o-P h o t o n ~ L a b o r a t o r y, ~ F a c u l t y ~ o f ~ I n f o r m a t i o n ~}$ \\ Technology, Pázmány Péter Catholic University, Budapest 1083, Hungary; ${ }^{\mathrm{d}}$ Mines Saint-Etienne, \\ Centre CMP, Département BEL, F - 13541 Gardanne France
}

\begin{abstract}
Functional mapping of the connectivity of the brain is an essential procedure for neurosurgeons aiming for maximal resection of a brain tumor, while minimizing damage to the eloquent cortex. Being able to delineate the location of critical cortical areas in individual patients allows the surgeon to preserve sensorimotor and cognitive functions. Several methods exist for functional mapping, however, none are capable of delivering fast, label free mapping of the cortex with millimeter precision. In this work, we investigate the potential use of infrared neural stimulation $(\lambda=1470 \mathrm{~nm})$ as a means of focal stimulation of the mouse cortex in vivo. Here, we show that the application of infrared neural stimulation induces in vivo cortical intracellular calcium signals in Layer II/III mouse neurons, using intravital calcium imaging with the genetically encoded calcium indicator GCaMP6f.
\end{abstract}

Keywords: Infrared neural stimulation, GECI, imaging, neuroscience.

\section{INTRODUCTION}

Direct electrical stimulation is a commonly used technique by neurosurgeons to map eloquent structures of the brain during surgeries like brain tumor resections. Having a precise and rapid way to map the brain during such procedures would allow surgeons to improve the extent of resection while preserving the eloquent and essential structures of the brain.

Infrared neural stimulation (INS) is a method of stimulating the brain by the exposure of tissue to pulsed infrared radiation at wavelengths that are strongly absorbed by water [1].As a result of a photothermal mechanism, neural responses are triggered with better spatial selectivity than electrical stimulation [2]. Up to now, INS has been widely applied and studied in the peripheral nervous system such as in the auditory system, or in the vestibular [3] and facial nerves [4], to give the beginning of a non-exhaustive list. In 2010, INS was applied for the first time in the central nervous system (CNS) by Cayce and colleagues in thalamocortical brain slices [5] before to show an inhibitory effect in rat somatosensory cortex [6], and an excitatory effect in non-human primates in the primary visual cortex [7], using optical intrinsic imaging or single unit electrophysiological recordings. More recently it was even used in combination with fMRI to map the mesoscale brain connectomes in cat and monkeys [8]. Calcium imaging was also used in vivo with simultaneous infrared brain stimulation in rodents with the synthetic indicator Oregon Green 488 BAPTA-1 AM to transiently label cortical astrocytes and neurons [9]. Thereafter, infrared pulses of $250 \mu \mathrm{s}$ were delivered at a frequency of $200 \mathrm{~Hz}$ for $500 \mathrm{~ms}$ and subsequent intracellular calcium signals were observed in cortical astrocytes and apical dendrites on the brain surface.

Here we show that INS can be used to evoke intracellular calcium signals, down to Layer $2 / 3$ neurons in the mouse cortex in vivo. Infrared pulses of $250 \mu$ s delivered in a train at $200 \mathrm{~Hz}$ for a duration of $500 \mathrm{~ms}$ were found to initiate calcium signals in cortical neurons of mice expressing GCaMP6f, as shown by two-photon in vivo microscopy. We also show that by increasing the amount of energy delivered locally to the mouse brain resulted in an increased number of neurons exhibiting calcium signals above a determined threshold.

*david.moreau@emse.fr; phone +33 (0)4 426168 77; 


\section{MATERIAL AND METHODS}

\subsection{Mice}

All experimental protocols were approved by the Animal Care and Experimentation Committee of the Institute of Experimental Medicine of the Hungarian Academy of Sciences (approval reference numbers PEI/001/194-4/2014 and PEI/001/1771-2/2015). All procedures complied with Hungarian and European regulations for animal research. All the procedures were performed on C57BI/6J (N=5 males, RRID:IMSR_JAX:000664) or Thy-1-Cre (N=10 males, RRID: IMSR_JAX:006143_JAX:010908) mice (P60-120). Animals had free access to food and water and were maintained in temperature-, humidity-, and light-controlled conditions.

\subsection{Surgical Procedure}

The surgical procedure was similar to the one described previously, adjusted with some minor modifications. As a summary, mice were anesthetized with a mixture of midazolam, fentanyl, and medetomidine ( $5 \mathrm{mg}, 0.05 \mathrm{mg}$ and 0.5 $\mathrm{mg} / \mathrm{kg}$ body weight, respectively). Finally, a circular craniotomy was made over the V1 region using a dental drill, and was fully covered with a cover glass.

\subsection{AAV labeling}

The injection procedure was performed as described previously [10], adjusted with some minor modifications. A hole of about $0.5 \mathrm{~mm}$ diameter was opened in the skull with the tip of a dental drill over the V1 cortical region (centered $2.5 \mathrm{~mm}$ lateral and $3.0 \mathrm{~mm}$ posterior to the bregma). Injections were made with a glass micro-pipette (tip diameter $\approx 10 \mu \mathrm{m})$ which were back-filled with $0.4 \mu \mathrm{l}$ vector solution $(\approx 6 \times 1013$ particles $/ \mathrm{ml})$. The solution was then injected slowly $(10 \mathrm{nl} / \mathrm{s}$ for first $30 \mathrm{nl}$, and $0.5 \mathrm{nl} / \mathrm{s}$ for the remaining quantity) into the cortex, at a depth of $450 \mu \mathrm{m}$ under the pia. For population imaging, we used AAV9.Syn.GCaMP6f.WPRE.SV40 or AAV9.Syn.Flex.GCaMP6f.WPRE.SV40 (in the case of Thy-1Cre); both viruses were from Penn Vector Core, Philadelphia, PA and are neuron-specific31. The cranial window was implanted over the injection site 2 weeks after the injection, as described in the surgical procedure section.

\subsection{Optical fiber preparation}

A multimode laser diode (LU1470T015, Lumics) with a fibered output (core diameter $105 \mu \mathrm{m}$, numerical aperture 0.15) was used to provide $1470 \mathrm{~nm}$ infrared radiation. The module temperature was controlled using the appropriate cooling block (LU_CB_T_0, Lumics). The optical fiber was placed into a custom-built guide cannula and mounted onto the arm of a micromanipulator (LN Junior LR, Luigs-Neumann, Ratingen, Germany).

\subsection{In vivo calcium imaging data acquisition}

After a brief sedation by $2 \%$ isoflurane, the animal was placed on a heating pad to maintain its' temperature at $38^{\circ} \mathrm{C}$, and its head was fixed by screwing the head-fixed metal bar to the custom mouse holder in the microscope setup. During the recording procedure, the anesthesia was maintained with a $0.5 \%$ isoflurane and carbogen mixture. An ultrasound transmission gel (AquaUltra Clear, Ultragel Hungary 200 Ltd, Hungary) was applied as an immersion medium. The mounted optical fiber was positioned underneath a 16X objective (Nikon LWD 16x/0.8 NA) under IR camera guidance. Switching to two-photon mode, a reference z-stack of the volume was acquired on a dual-scanhead two-photon microscope (FemtoS-Dual, Femtonics Ltd, Budapest, Hungary) equipped with a femtosecond pulsed laser tuned to 910 nm (Chameleon Ultra II, Coherent, Santa Clara, California). A single acquisition plane was selected and full-frame imaging was started in resonant scanning mode at $30.9375 \mathrm{~Hz}$. The control of calcium signal recording and the trigger of infrared stimulation was done using the microscope's acquisition software (MESc). In order to have a better incident infrared radiation angle, the head of the mouse was tilted with respect to the optical axis by about $20^{\circ}$.

\subsection{Data analysis}

Fluorescent time series depicting the calcium changes occurring in vivo in both the neuropil and the selected cells were analyzed with the MESc data acquisition software and the MES curve analyzer tool. Briefly, regions of interest were manually selected to coincide with single cell somata. The raw fluorescence calcium traces were extracted to Excel and analyzed using custom Matlab scripts. They were transformed to show fluorescence change according to the formula: 


$$
\frac{\Delta F}{F_{0}}=\frac{\left(F-F_{0}\right)}{F_{0}}
$$

where $\mathrm{F}$ is the fluorescence at any given point in time, and $\mathrm{F} 0$ is the mean fluorescence value for the 3000 to $4500 \mathrm{~ms}$ range, preceding the infrared stimulus presentation. Following this, the signal-to-noise ratio (SNR) is:

$$
S N R=\left(\frac{\Delta F}{F_{0}}\right)_{\text {peak }} / \sigma F
$$

where $\sigma \mathrm{F}$ is the standard deviation of the baseline period. We took those events as a Calcium response that exceeded three times the standard deviation of the baseline period.

\subsection{Infrared light stimulation}

The power supply (LU_DR_AD, Lumics) allowed control of both the exposure time and the power of the infrared light delivered to the biological tissue. It was externally controlled by a waveform generator (3390 waveform generator, Keithley) triggered by MESc acquisition software. Average power was measured using a S145C integrating sphere photodiode power sensor attached to the PM100D power meter (Thorlabs) from the tip of the fiber after the completion of each experiment, to be sure that no damage was done to the tip of the fiber. Energy density of infrared pulses was calculated as the energy density delivered by the tip of the fiber to avoid any approximation in the calculation (diameter $=105 \mu \mathrm{m})$. The temporal profile of the infrared stimulation consisted of $250 \mu \mathrm{s}$ pulses applied at $200 \mathrm{~Hz}$ during $500 \mathrm{~ms}$.

\section{RESULTS}

\subsection{Infrared exposure induces transient calcium signals in single neurons}

Our initial experiments aimed to demonstrate that pulsed infrared light trains were able to evoke intracellular calcium signals within Layer 2/3 neurons in mouse cortex in vivo. We acquired two-photon calcium fluorescence images during a total period of 30 seconds and applied infrared neural stimulation 5 seconds after the beginning of the recordings, to be able to get a fluorescence baseline. We performed this protocol 5 times in a recording loop, in order to assess the reproducibility of the response and to have an average response, to have a better the signal-to-noise ratio in the calcium signal recorded. In the following example, the pulse energy density at the tip of the fiber was $2.19 \mathrm{~J} \cdot \mathrm{cm}^{-2}$. The depth of the cells imaged in the field of view was determined thanks to the z-stack and found to vary from about $150 \mu \mathrm{m}$ till 300 $\mu \mathrm{m}$ under the brain surface as a result of the tilting of the animal head, corresponding to the location of Layer $2 / 3$ neurons.

Figure 1 shows the evolution of two different types of signals over time could be observed across all of the neural cells: neurons responding to the infrared exposure by an increase in the GCaMP6f fluorescence, and neurons presenting timecourses without any infrared induced response.
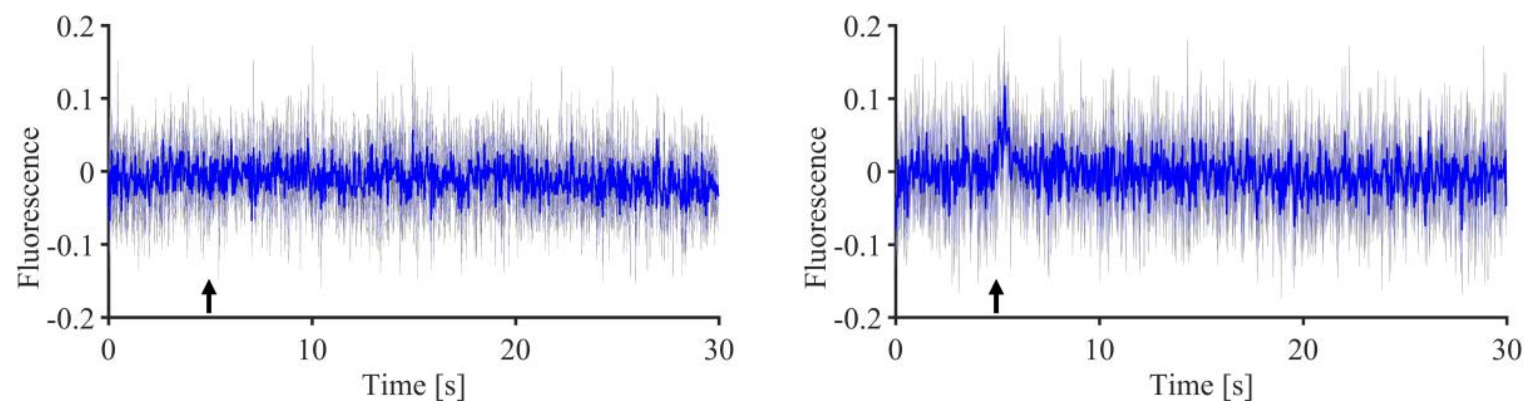

Figure 1. Left. Example of 5 recordings average GCaMP6f fluorescence timecourse in a single neuron not responding to the infrared exposure. Right. Example of 5 recordings average GCaMP6f fluorescence timecourse in a neuron responding to the infrared exposure. In blue: the average of the repetition of the five recordings reproduced with the same infrared exposure

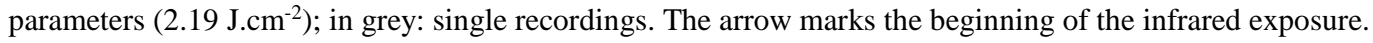




\subsection{Energy density affects neurons responses}

Given the capability of pulsed infrared light trains to evoke intracellular calcium ion concentration changes in single neurons, we swept the infrared power and thus the energy density delivered, to try to find stimulation and damage thresholds, as well as the relationship between calcium signals generation and the energy density delivered. We applied Four different pulse energy densities: $1.33 \mathrm{~J} \cdot \mathrm{cm}-2,1.76 \mathrm{~J} \cdot \mathrm{cm}-2,2.19 \mathrm{~J} \cdot \mathrm{cm}-2$ and 2.63 $\mathrm{J} \cdot \mathrm{cm}-2$. We performed experiments with the same principle of 5 recordings in a loop to show reproducibility of single neuron responses and to improve the signal to noise ratio of our dataset. Figure2 shows that there is an increasing number with respect to energy density of responding neurons registered at the two-photon imaging plane. Interestingly, we can also notice that if some cells are activated for each condition, cells active in low energies conditions are not necessarily an overlapping population with those in the high-energy conditions.
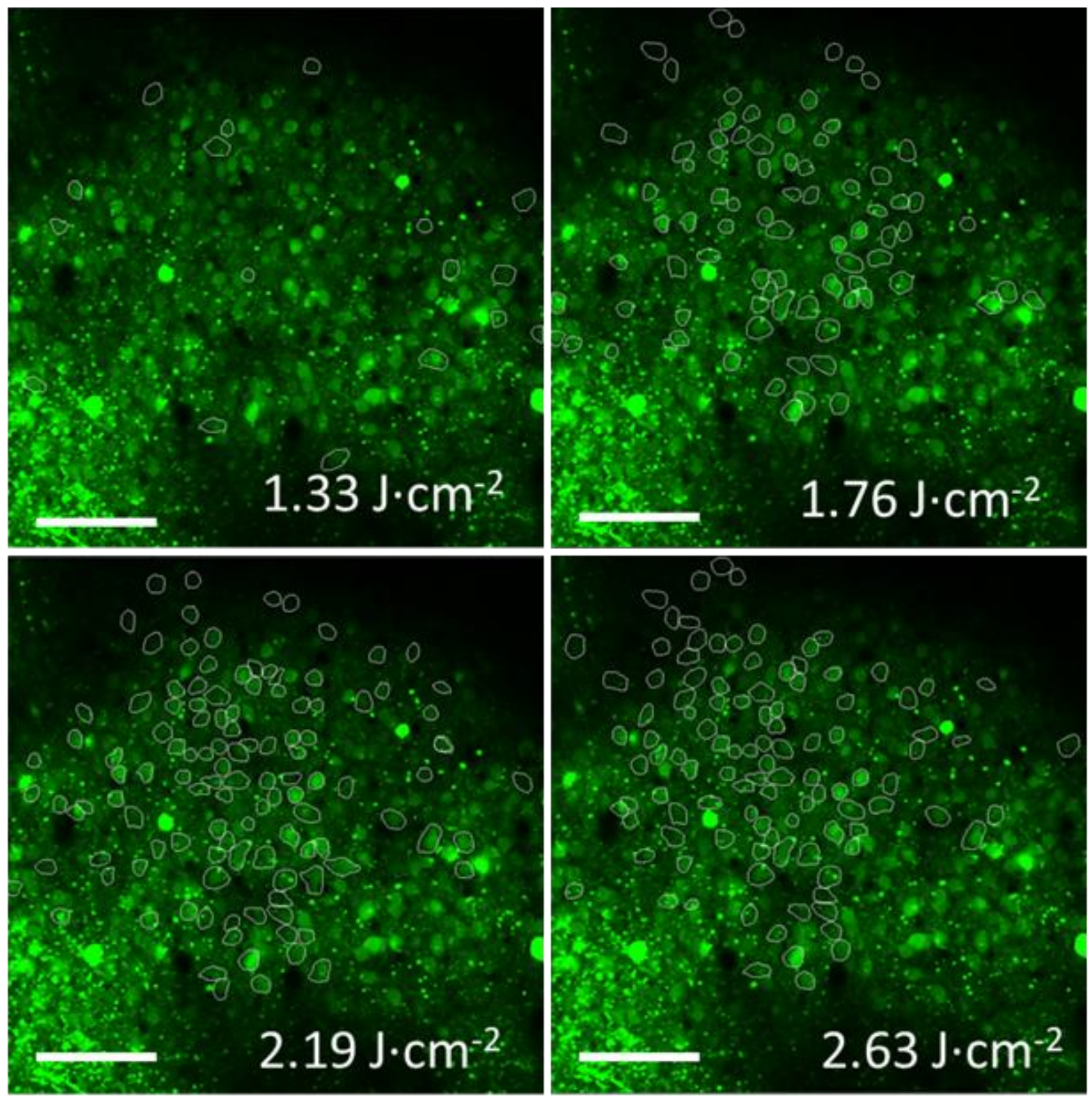

Figure 2. Representative two-photon field of view of a single mouse visual cortex showing responsive identified neurons. 


\subsection{Brain damage assessment}

Infrared neural stimulation being mediated by a local photothermal mechanism, there is a concern about potential thermally induced damage in the targeted tissue. During the recording sessions, in order to measure the appearance of damage in real-time, we assessed the autofluorescence level at the surface of the brain, since this area is the first target of possible damage [11]. Afterwards, we also confirmed what was observed during the recording sessions by studying the evolution of the value of $F_{0}$ in each neuron imaged over time and stimulation performed. Figure 3 shows this average evolution in all the cells imaged in this study. We can see that repeated infrared stimuli don't affect the GCaMP6f baseline in the targeted neurons for the energy densities used, up to an energy density of $2.63 \mathrm{~J} . c m^{-2}$, which is identified here as the highest energy density before to reach damage threshold, (application of $2.84 \mathrm{~J} . \mathrm{cm}^{-2}$ resulted in damage, data not shown).
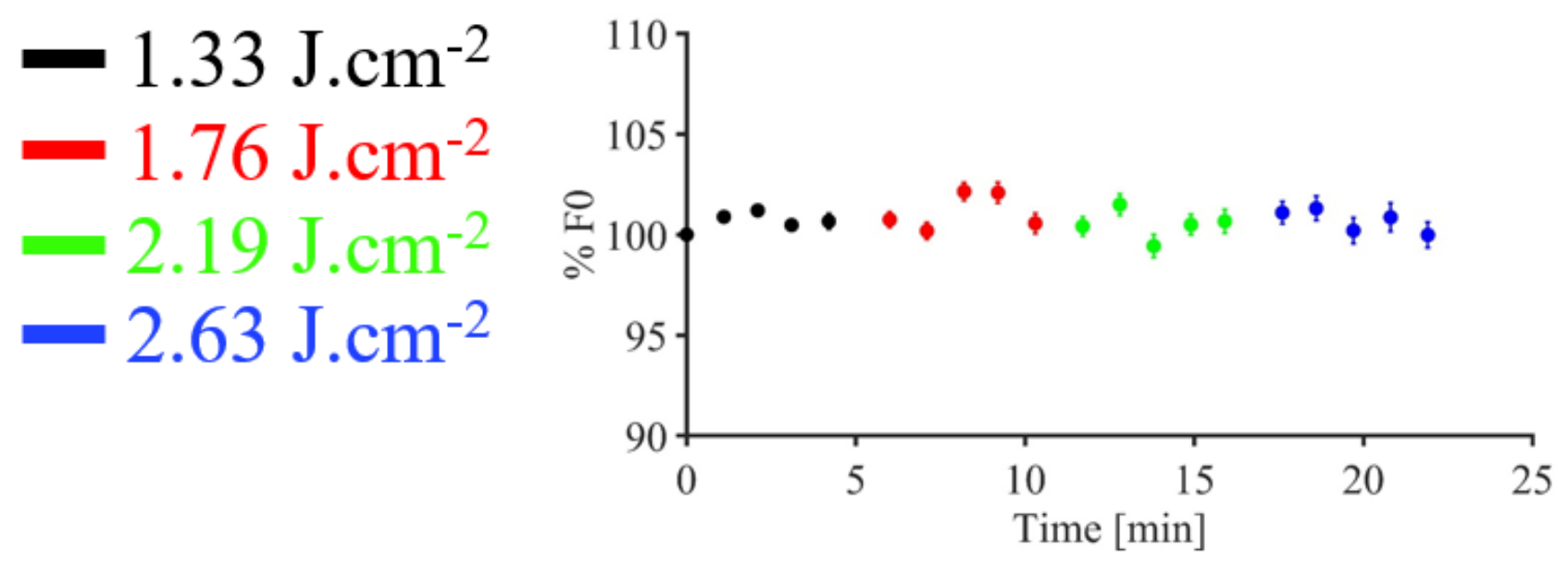

Figure 3. Evolution of the value of $\mathrm{F}_{0}$ in all imaged neurons over time and stimulation performed

\section{CONCLUSION}

Here we showed that INS can be used to evoke calcium signals in cortical layer II-III neurons in vivo with two-photon imaging of mice expressing GCaMP6f. Infrared pulses of $250 \mu$ s repeated 100 times at $200 \mathrm{~Hz}$ elicited reproducible and damage-free calcium signals in single neurons up until the damage threshold found to be $2.84 \mathrm{~J}_{\mathrm{cm}}{ }^{-2}$. An increasing number of responding neurons with respect to energy density was observed. If some of them are activated for each condition, cells active in low energies conditions are not necessarily an overlapping population with those in the highenergy conditions.

However, within the field of view, no evident spatial selectivity could be identify without further analysis. In order to have an extended view to be able to study the spatial selectivity of such stimulation in the brain, future work will involve combining imaging and electrophysiological recording, with implantation of custom made transparent, flexible and biocompatible microelectrode arrays [12].

\section{REFERENCES}

[1] Wells, J., Kao, C., Jansen, E. D., Konrad, P., and Mahadevan-Jansen, A., “Application of infrared light for in vivo neural stimulation,” J. Biomed. Opt., 10 (6), 064003, (2005).

[2] Izzo, A. D., Suh, E., Pathria, J., Walsh Joseph T., J., Whitlon, D. S., and Richter, C.-P., "Selectivity of neural stimulation in the auditory system: a comparison of optic and electric stimuli," J. Biomed. Opt., 12 (2), $21007-$ 21008, (2007).

[3] Rajguru, S. M., Rabbitt, R. D., Matic, A. I., Highstein, S. M., and Richter, C.-P., "Selective activation of vestibular hair cells by infrared light," Biophys. J., 98 (3), 507a, (2010).

[4] Teudt, I. U., Nevel, A. E., Izzo, A. D., Walsh, J. T., and Richter, C., "Optical stimulation of the facial nerve: a new monitoring technique?," Laryngoscope, 117 (9), 1641-1647, (2007). 
[5] Cayce, J. M., Kao, C. C., Malphrus, J. D., Konrad, P. E., Mahadevan-Jansen, A., and Jansen, E. D., "Infrared Neural Stimulation of Thalamocortical Brain Slices," IEEE Journal of Selected Topics in Quantum Electronics, 16 (3). 565-572, 2010, doi: 10.1109/JSTQE.2009.2032424.

[6] Cayce, J. M., Friedman, R. M., Jansen, E. D., Mahavaden-Jansen, A., and Roe, A. W., "Pulsed infrared light alters neural activity in rat somatosensory cortex in vivo," Neuroimage, 57 (1), 155-166, (2011).

[7] Cayce, J. M., Friedman, R. M., Chen, G., Jansen, E. D., Mahadevan-Jansen, A., and Roe, A. W., "Infrared neural stimulation of primary visual cortex in non-human primates," Neuroimage, 84, 181-190, (2014).

[8] Xu, A. G., Qian, M., Tian, F., Xu, B., Friedman, R. M., Wang, J., Song, X., Sun, Y., Chernov, M. M., Cayce, J. M., Jansen, E. D., Mahadevan-Jansen, A., Zhang, X., Chen, G., and Roe, A. W., "Focal infrared neural stimulation with high-field functional MRI: A rapid way to map mesoscale brain connectomes," Sci. Adv., 5 (4), eaau7046, (2019).

[9] Cayce, J. M., Bouchard, M. B., Chernov, M. M., Chen, B. R., Grosberg, L. E., Jansen, E. D., Hillman, E. M. C., and Mahadevan-Jansen, A., "Calcium imaging of infrared-stimulated activity in rodent brain," Cell Calcium, 55 (4), 183-190, (2014).

[10] Szalay, G., Judak, L., Katona, G., Ocsai, K., Juhasz, G., Veress, M., Szadai, Z., Feher, A., Tompa, T., Chiovini, B., Maak, P., and Rozsa, B., "Fast 3D Imaging of Spine, Dendritic, and Neuronal Assemblies in Behaving Animals," Neuron, 92 (4), 723-738, (2016).

[11] Chernov, M. M., Chen, G., and Roe, A. W., "Histological assessment of thermal damage in the brain following infrared neural stimulation," Brain Stimul., 7 (3), 476-482, (2014).

[12] Donahue, M. J., Kaszas, A., Turi, G. F., Rózsa, B., Slézia, A., Vanzetta, I., Katona, G., Bernard, C., Malliaras, G. G., and Williamson, A., "Multimodal Characterization of Neural Networks using Highly Transparent Electrode Arrays," eNeuro, ENEURO-0187, (2018). 